Ethik Med 2008 · 20:83-85 DOI 10.1007/s00481-008-0562-6 ○ Springer Medizin Verlag GmbH 2008
Markus Zimmermann-Acklin · Luzern

\section{Mit Helium in den Tod? Zur Diskussion um die Beihilfe zum Suizid in der Schweiz}

ie schweizerische Sterbehilfeorganisation Dignitas führt seit einiger Zeit Sterbebegleitungen mit Hilfe von Heliumgas durch. Der Sterbewillige zieht sich mit Hilfe eines Sterbehelfers eine Atemmaske über Mund und Nase, atmet Helium ein, wird bewusstlos und verstirbt. Das Gas verdrängt den Sauerstoff aus der Lunge, was zum Erstickungstod führt. Der Vorgang wird gefilmt und der Film post mortem der Staatsanwaltschaft als Beleg für die Tatherrschaft des Sterbewilligen übergeben. Dieses Prozedere erlaubt das Umgehen einer - je nach Sichtweise - lästigen bzw. wichtigen Hürde, nämlich des Einbezugs einer Ärztin, welche den Sterbewilligen untersucht, den Sterbewunsch überprüft und unter Umständen ein todbringendes Mittel - gewöhnlich das Natriumpentobarbital, kurz NaP - verschreibt.

Über die Beihilfe zum Suizid wird gegenwärtig in vielen europäischen Ländern debattiert. Ausgangspunkt sind in der Regel besonders berührende Einzelbeispiele: Der aufgrund einer Attacke von Rechtsextremen tetraplegische Noël Martin in Deutschland, die an einem Gesichtskrebs erkrankte und inzwischen mit $\mathrm{NaP}$ aus dem Leben geschiedene Französin Chantal Sébire, der an Muskelschwund leidende und durch den Abbruch der künstlichen Beatmung in Italien verstorbene Piergiorgio Welby oder die nach vergeblichen Bemühungen um die Gewährung der Beihilfe zum Suizid gestorbene ALS-Patientin Diane Pretty in England. Zudem entschied im Februar in Luxemburg eine Parlaments- mehrheit in erster Lesung zugunsten eines Gesetzes, welches die Strafbefreiung der Tötung auf Verlangen und der Suizidbeihilfe vorsieht [3].

In der Schweiz verliefen die Debatten über Praxis und Regelung der Suizidbeihilfe bislang relativ ruhig. Das ändert sich gegenwärtig: Die Anwendung unkonventioneller Suizidmethoden wie die Verwendung von Helium oder Plastiksäcken, das Ausstellen von NaP-Rezepten von pensionierten Ärzten, die keine Bewilligung mehr dazu haben, die Beihilfe bei psychisch Kranken und Sterbewilligen, die für den Suizid aus dem Ausland anreisen und aufgrund von Widerstand der Anwohner nicht mehr in Wohnungen, sondern in Industriegebäuden und auf Parkplätzen ihrem Leben ein Ende setzen, haben zu einer markanten Verschärfung der öffentlichen Auseinandersetzungen geführt. Die Frage nach gesetzlichen Einschränkungen der bestehenden „wilden Praxis“ beschäftigt die eidgenössische Regierung, das Parlament, ärztliche Fachgesellschaften, Spitalleitungen, Kirchenvertreter und aufgebrachte Bürgerinnen und Bürger genauso wie die Nationale Ethikkommission und die Zentrale Ethikkommission der Schweizerischen Akademie der Medizinischen Wissenschaften.

In diesen Konflikten erscheint die Ärzteschaft besonders exponiert: Während große Teile der Gesellschaft (und auch des Parlaments) die ärztliche Suizidbeihilfe als Option grundsätzlich zur Verfügung haben möchten und sich gegen ein Verbot aus- 
sprechen, wehren sich ärztliche Standesvertretungen gegen eine Beteiligung, da sie die Beihilfe als unvereinbar mit dem ärztlichen Standesethos ansehen. Doch: Je stärker die Ärzteschaft sich in die Debatten einschaltet, desto mehr gerät sie unfreiwillig in die Rolle von Experten in einem Bereich, der weit über die Medizin hinausgeht [1].

Dieses Phänomen wird besonders deutlich angesichts der Beihilfe bei psychisch Kranken. Das schweizerische Bundesgericht hat kürzlich als Antwort auf eine Klage, welche die rezeptfreie Abgabe des $\mathrm{NaP}$ für einen psychisch Kranken zum Gegenstand hatte, festgehalten, dass es kein Recht auf Beihilfe zum Suizid gebe und die Rezeptpflicht für das todbringende Mittel und damit die ärztliche Untersuchungspflicht aus rechtlichen Gründen aufrecht erhalten werden müsse [4]. Der Kläger vertrat einen psychisch Kranken, 1953 geborenen Mann, der bereits zwei Suizidversuche hinter sich hatte und keinen Arzt finden konnte, der bereit war, ihm das $\mathrm{NaP}$ zu verschreiben. Das Ziel der Klage bestand darin, der Sterbehilfegesellschaft Dignitas zu ermöglichen, auch ohne ärztliche Verschreibung die nötigen $15 \mathrm{~g} \mathrm{NaP}$ beziehen zu können. Im Urteil hält das Bundesgericht jedoch an der Rezeptpflicht fest und betont, wie heikel solche ärztlichen Entscheidungen insbesondere bei psychisch Kranken seien, und verlangt überdies für derartige Situationen ein vertieftes psychiatrisches Fachgutachten.

Dass die Fachgesellschaft der Psychiater hinsichtlich der Ausstellung solcher Gutachten sogleich ablehnend reagiert hat, ist wenig erstaunlich: Geht es in Fällen wie diesen doch nicht um Sterbende, die in ihrer letzten Lebensphase ihre Ärztin darum bitten, sie in ihrer Not nicht im Stich zu lassen, sondern um Menschen, die mitten im Leben stehen und bei welchen therapeutische Maßnahmen auf die Verhinderung von Suizidalität ausgerichtet sind. Die Psychiater weisen zu Recht darauf hin, dass eine Gesellschaft, welche für solche Situationen eine Option zur Suizidbeihilfe schaffen bzw. nicht verbieten möchte, diese Aufgabe nicht an die Ärztinnen und Ärzte delegieren könne.

Dieses Problem, ärztliche Kompetenz in einem Bereich einzufordern, der außerhalb der medizinischen Aufgaben liegt, ist auch in den Niederlanden manifest [5] und wiederholt sich in der Debatte um eine angemessene rechtliche Regelung der offenkundig bestehenden Probleme in der Schweiz [2,6]. Die bislang diskutierten Vorschläge weisen - abgesehen von der grundsätzlichen Bewertung der Suizidbeihilfe - aus ethischer Sicht mindestens zwei Schwierigkeiten auf: Erstens sollen Ärztinnen und Ärzte mit der Entscheidung darüber, wer sich das Leben nehmen darf und wer nicht, eine Kompetenz erhalten, die sie zumindest außerhalb der unmittelbaren Sterbephase aus guten Gründen nicht übernehmen wollen. Zweitens wird klar, dass mit einer gesetzlichen Regelung Grauzonen nicht abgeschafft, sondern lediglich verschoben werden. Bestehende Unsicherheiten werden durch neue ersetzt, wenn es beispielsweise nötig wird, die Suizidbeihilfe bei Kindern, Jugendlichen, psychisch Kranken, existentiell Leidenden, Vereinsamten, an Demenz Erkrankten oder bei Doppelsuiziden gesetzlich zu regeln. Die Urteilsfähigkeit alleine kann als Kriterium nicht genügen, wenn der Gesetzgeber gleichzeitig die staatliche Pflicht zum Lebensschutz gewährleisten möchte.

Meines Erachtens soll hier etwas geregelt werden, was sich in letzter Konsequenz einer Regelung entzieht. Die Entscheidung, sich das Leben zu nehmen, hat neben allem Nachvollziehbaren stets auch irrationale, verwirrende und aufschreckende Anteile. Wie kann eine solche Entscheidung, die auf das menschliche Umfeld in der Regel verstörend wirkt, nach Maßstäben der Qualitätskontrolle geregelt bzw. „normalisiert" werden? Entschließt sich ein Mensch aufgrund seiner Isolation oder der Ablehnung, in ein Pflegeheim zu ziehen, zum Suizid, dann besteht die angemessene Reaktion darin, die Aufmerksamkeit füreinander zu stärken und die Pflegequalität in 
den Heimen zu sichern. Dagegen kann eine gesetzliche Regelung, welche die Qualität der Suizidbegleitung absichern soll, indem die Sterbehilfegesellschaften einen Zertifizierungsprozess durchlaufen müssen, zwar den Einsatz von Helium und Plastiksäcken verhindern, ist aber gleichzeitig Ausdruck von Resignation. Soll der Suizid - begleitet oder nicht - als Ausweg aus schwierigen Lebenssituationen salonfähig gemacht werden? Soll aus einer nachvollziehbaren Grenzüberschreitung in extremen Ausnahmefällen bei sterbenden Patienten eine Standardentscheidung werden? Die Gefahr bestünde dann weniger in möglichem Missbrauch als in einer Vernachlässigung der Fürsorgepflichten, die Folge wäre eine Banalisierung des Sterbens.

\section{Anschrift}

\section{Dr. theol. Markus Zimmermann-Acklin}

Institut für Sozialethik

Universität Luzern

Gibraltarstraße 3, Postfach 7766

6000 Luzern 7, Schweiz

E-Mail: markus.zimmermann@unilu.ch

\section{Literatur}

1. Bosshard G, Broeckaert B, Clark D, Materstvedt LJ, Gordijn B, Müller-Busch HC (2008) A role for doctors in assisted dying? An analysis of legal regulations and medical professional positions in six European countries. J Med Ethics 34:28-32

2. Brunner $A$ (2006) Skizze für ein Gesetz betreffend organisierte Suizidhilfe. In: Rehmann-Sutter C, Bondolfi A, Fischer J, Leuthold M (Hrsg) Beihilfe zum Suizid in der Schweiz. Beiträge aus Ethik, Recht und Medizin. Lang, Bern, S 247-253

3. Gillen E, Jacobs F (2008) Zwischen den Gesetzen. Zur Euthanasie-Diskussion im Großherzogtum Luxemburg (März 2008). Hessisches Ärzteblatt 69:328-329

4. Schweizerisches Bundesgericht (2006) BGE 133 I 58

5. van der Heide $A$, Onwuteaka-Philipsen $B D$, Rurup $M L$ et al (2007) End-of-Life practices in the Netherlands under the Euthanasia Act. N Engl J Med 356:1957-1965

6. Venetz $P$ (2008) Suizidhilfeorganisationen und Strafrecht. Schulthess, Zürich Basel Genf 